\section{BOSNIAN REGISTRY OF THERAPEUTIC CARDIAC CATHETERISATION}

S. Dinarević, Z. Begić, M. Halimić

Pediatric Clinic, Clinical Centre of University Sarajevo, Bosnia and Herzegovina, Sarajevo, Bosnia-Herzegovina

Introduction: Cardiac catheterization (CC) in treatment of congenital heart disease (CHD) nowdays has much more therapeutic, then diagnostic importance. In Bosnia and Herzegovina post war CC is in process of development.

Aim: To present a review of Bosnian registry of cardiac therapeutic catheterization cases.

Subjects and methods: Patients with CHD during May 1998- February 2010 hospitalised at Paediatric clinic were included. An evaluation of pts was conducted by history, physical examination, chest X-ray, electrocardiograms and Colour Doppler echocardiogram. The need forcardiaccatheterization was primarily determined by paediatric cardiologist or suggested by paediatric cardiac surgeon.

Results: 180 cardiac catheterizations were performed. Diagnostic catheterization was done in $155(86 \%)$ and therapeutic in $25(14 \%)$ patients, with age ranging from 2 days to 21 years, mean 5,3 years. $53 \%$ pts were females. Indications for therapeutic catheterizations were:pulmonary valve stenosis 11 (44\%), patent ductus arteriosus (PDA) $8(32 \%)$, aortic valve stenosis (AS), coarctation of aorta $3(12 \%)$, atrial septal defect closure $2(8 \%)$, one extraction of foreign body from right ventricle. CC team did five successful balloon atrial septostomy. Duration of the performing catheterization lasted for PDA 13', PS 8,4' and AS 10,3'. Complication was: coil to the right branch of pulmonary artery (2), unsuccessful pulmonary valve balloon dilatation with displastic valve (1), airway spasm during anethesia (1).

Conclusion: A limited experience with cardiac catheterisation in patients presenting with $\mathrm{CHD}$ has been reported in an attempt to highlight the importance of advancements in the use of this technology in Bosnia and Herzegovina.

Keywords: Therapeutic cardiac catheterization, congenital heart disease

\section{SPORTS HEART IN CANTON SARAJEVO}

\section{S. Dinarević ${ }^{1}$, M. Kulić2 ${ }^{2}$ A. Kreso ${ }^{3}$, I. Poplata ${ }^{1}$}

${ }^{1}$ Paediatric Clinic Clinical University Centre of Sarajevo, ${ }^{2}$ Centar for Heart Sarajevo, ${ }^{3}$ P.I. for Sport Medicine of Canton Sarajevo, Sarajevo, Bosnia-Herzegovina

Due to possible risk of sudden death during sport activities, screening of competitive sportists is of vital importance. The aim of this study is to evaluate the incidence of heart diseases by cardiovascular screening of young sportists as well as to establish preventive strategy. The research was conducted during 2007-2009. Study formed 281 sportists (245 boys), age 8-18 years, mean age 15.26 years. In order to analyse echocardiographic parametersa, sportists were divided into 5 age groups according to body weight. After evaluation of history (familial, personal and cardiac), measurement of body weight and height, blood preassure, pulse rate, oxygen saturation was performed as well as 12 leads ECG, specialist examination (paediatrician, specialist of sport medicine and paediatric cardiologists) with echocardiography. Results of study showed that all participants were free of symptoms. No congenital heart anomalies potentialy lethal for young sportists was diagnosed, $0 \%$ sportists had cardiovascular abnormalities which would require further evaluation, or had positive cardiac history, abnormal auscultatory findings, hypertension, or abnormal ECG. Ehocardiographically mass of left vetricle in 5 age groups in relation to body weight was significantlly enlarged $(P>0.0001)$ as a part of normal findings of heart adaptation on excercisesyndrom of sports heart.

Conclusion: It is needed to establish adequate strategy of cardiovascular screening of young sportist with the aim of minimalisation/prevention of possible risk of sudden death. 